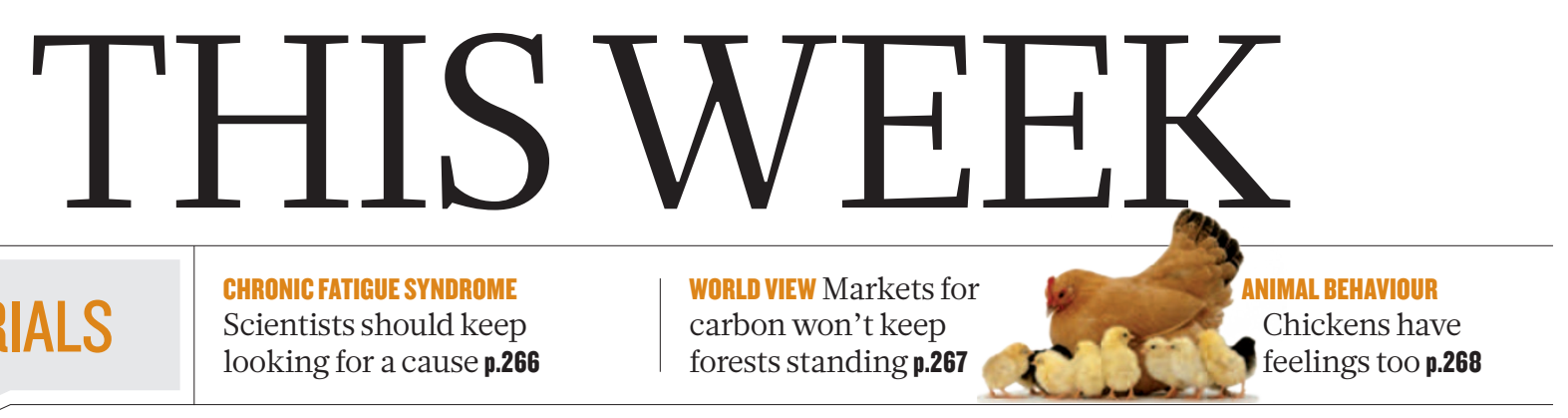

\title{
A bold unifying leap
}

\section{How James Clerk Maxwell made a difference 150 years ago.}

$\mathrm{W}$ hat is it that makes physicists proud to be physicists? One answer lies in James Clerk Maxwell's equations. Physicists can rejoice in a historical moment of great insight, can share in the expressions of that insight that only they can understand in any depth, can bond over surviving the didactic stress that many students experience in learning to apply them and, above all, can roam freely in deploying the power thus provided for understanding the world and, on occasion, changing it.

These communal consequences of a grand discovery might arise in any scientific discipline. But Maxwell's equations are particularly characteristic of physics in the way in which they unified previously disparate laws and provided a foundational framework for vast industries - both academic and commercial.

In this issue we celebrate the first expression of those equations by Scottish physicist Maxwell in the Philosophical Magazine 150 years ago. There he drew together several strands of understanding about the behaviour of electricity, of magnetism, of light, and of the ways in which these fundamental aspects of nature behave in matter. As Albert Einstein remarked, "so bold was the leap" of this work that it took decades for physicists to grasp its full significance. And although it was a wonderful expression of science at its purest, it was forged in the thoroughly practical culture of intellects at that time (see page 289).

By the time Maxwell began this work, it was known that a pattern of electric fields could be related to a distribution of electric charges; that single magnetic charges, in contrast, do not exist; that a moving electrical charge also generated a magnetic field; and that a time-varying magnetic field generated an electrical field.

One of Maxwell's achievements was the insight that magnetic fields can be electrically generated by varying electric fields, as well as by travelling charges. In expressing this and the established rules in a set of equations, he provided a framework that revolutionized our ability to understand and apply electricity and magnetism in disciplines ranging from astronomy to biology to telecommunications.

In materials, the application of Maxwell's equations depends on an understanding of the way in which those materials affect the propagation of electric and magnetic fields. In some crystals and in Earth's atmosphere, for example, electromagnetic waves propagate differently in different directions, and may also, on entering the medium, be split into components that propagate differently. Yet all are described by Maxwell's equations. An intriguing application today is in metamaterials, which have unnatural propagation properties that enable the development of extraordinarily powerful lenses and optical cloaking (see page 292).

It is not only in materials that these equations can be applied. Empty space was also illuminated by Maxwell. His identification of the generation of magnetic fields by changing electric fields, coupled with the known generation of electric fields by changing magnetic fields, brought the insight that waves combining electricity and magnetism might travel in a self-sustaining way. In subsequent work, he concluded that light was just such a wave. He identified a single

propagation velocity in empty space of all electromagnetic radiation - known in his time as the speed of light, now also known to apply to radio, infrared and ultraviolet waves, to $\mathrm{X}$-rays and $\gamma$-rays.

That speed is determined in his equations by two characteristics of the medium in which the waves propagate - the electrical permittivity and the magnetic permeability. These have particular values in

"It is for Maxwell's insights in electromagnetism that scientists in all disciplines can be most grateful." to realise that the speed of light in empty space is not only a constant regardless of direction but also a constant no matter where the observer is and no matter how the observer is moving.

It is due to the ever-more-unifying discoveries of Maxwell's successors, and recent, often controversial, hypotheses in that spirit (see page 286), that much emphasis is today placed on that aspect of Maxwell's equations. Maxwell did other grand things in science - and this year is also the 150th anniversary of his taking the first true colour photographs. But it is for his unifying insights in electromagnetism that scientists in all disciplines can be most grateful, and physicists most proud.

\section{Into ignorance}

\section{Vote to overturn an aspect of climate science marks a worrying trend in US Congress.}

A s Nature went to press, a committee of the US Congress was poised to pass legislation that would overturn a scientific finding on the dangers of global warming. The Republicansponsored bill is intended to prevent the US Environmental Protection Agency (EPA) from regulating greenhouse-gas emissions, which the agency declared a threat to public welfare in 2009. That assessment serves as the EPA's legal basis for regulation, so repealing the 'endangerment finding' would eliminate its authority over greenhouse gases.

That this finding is scientifically sound had no bearing on the decision to push the legislation, and Republicans on the House of Representatives' energy and commerce committee have made clear their disdain for climate science. At a subcommittee hearing on 14 March, anger and distrust were directed at scientists and respected scientific societies. Misinformation was presented as fact, truth was twisted and nobody showed any inclination to listen to scientists, let alone learn from them. 